\title{
Estimación de la incertidumbre en un prototipo experimental basado en hardware libre para la medición de variables físicas que describen el movimiento de una partícula \\ Estimation of the uncertainty in an experimental prototype based on open hardware for the measurement of physical variables that describe the movement of a particle
}

Manuel R. Nevárez Toledo

Docente Investigador, Coordinador de Investigación, Responsable del Laboratorio de Investigación Tecnologías e Innovación. Escuela Ingeniería de Sistemas y Computación. Pontificia Universidad Católica del Ecuador Sede Esmeraldas. Esmeraldas. Ecuador.

manuel.nevarez@pucese.edu.ec

Verónica Yánez Ortiz

Docente titular auxiliar del Área de Matemáticas de la Escuela Ingeniería de Sistemas y Computación de la Pontificia Universidad Católica del Ecuador Sede Esmeraldas. Esmeraldas,

Ecuador.

veronica.yanez@pucese.edu.ec

Recepción: 07/12/2017. Aceptación: 09/01/2018. Publicación: 14/06/2018

Citación sugerida:

Nevárez Toledo, M. R. y Yánez Ortiz, V. (2018). Estimación de la incertidumbre en un prototipo experimental basado en hardware libre para la medición de variables fisicas que describen el movimiento de una partícula. $3 C$ Tecnología: Glosas de innovación aplicadas a la pyme, 7(2), 62-81. DOI: http://dx.doi.org/10.177993/3ctecno.2018. v7n2e26.62-81/ 


\section{RESUMEN}

La cinemática estudia el movimiento de una partícula sin considerar las causas que lo producen, mientras que la dinámica estudia los factores que producen alteraciones en un sistema. Se propone la creación de un equipo de medición que pueda registrar parámetros físicos enfocados en la cinemática y dinámica, utilizando las ecuaciones deducidas del cálculo para el movimiento, modificando: distancia del recorrido, ángulo de inclinación y masa de los cuerpos. La medición del tiempo servirá para obtener la incertidumbre provocada en los experimentos, la dispersión de los datos disponibles se relaciona con el tamaño de la muestra y el procedimiento utilizado. En las mediciones indirectas se emplearon ecuaciones diferenciales debido a la correlación en las variables físicas para el análisis de la incertidumbre. Uno de los métodos para el análisis de la incertidumbre se basa en el análisis estadístico, otra manera de estimarla es a través de la experiencia o información externa disponible. Los experimentos realizados en el plano inclinado para el estudio de la velocidad, aceleración y fuerza de fricción para ángulos mayores a $15^{\circ}$ presentaron un error relativo del $1.8 \%$ en la variable de tiempo, la incertidumbre estándar mantuvo una distribución normal con un nivel de confianza del $95 \%$.

\section{ABSTRACT}

The kinematic studies the motion of a particle without considering the effects that produce movement, while the dynamics studies the factors that produce changes in a system. It proposes the establishment of measuring equipment that can record physical parameters focused on the kinematics and dynamics, using the equations deducted from the calculation for the movement, by modifying travel distance, angle of inclination and mass of the objects. The measurement of the time will be used to obtain the uncertainty in the experiments, the dispersion of the available data is related to the size of the sample and the procedure used. In the indirect measurements were used equations due to the correlation in the physical variables for the analysis of uncertainty. One of the methods for uncertainty analysis is based on the statistical analysis, another way to estimate this is through experience or external information available. The experiments made in the inclined plane for the study of the velocity, acceleration and force of friction to angles greater than $15^{\circ}$, showed a relative error of $1.8 \%$ in the time variable, the standard uncertainty had a normal distribution with a confidence level of $95 \%$. 


\section{PALABRAS CLAVE}

Cinemática, Dinámica, Incertidumbre, Hardware libre, Medición.

\section{KEY WORDS}

Kinematics, Dynamics, Uncertainty, Open hardware, Measurement. 


\section{INTRODUCCIÓN}

Un experimentador tiene la tarea de medir magnitudes, lo que permite establecer nuevas leyes y verificar la validez de otras previamente establecidas. Estos resultados se expresan con un número y una unidad, dependiendo del patrón elegido. Las mediciones producen incertidumbres, ocasionadas por aparatos de medidas defectuosos o limitaciones, ya que el verdadero valor de una magnitud depende de las estimaciones o aproximaciones que, a su vez, dependen de la impericia del observador. En las mediciones se identifican tres elementos: número, unidad e incertidumbre, valores asignados mediante varias técnicas. Una de ellas corresponde a las cifras significativas, que dependen de la precisión del instrumento de medida, con los valores se realizan los cálculos que permiten obtener confianza en los resultados (U.L.P.G.C., 2017).

Las mediciones producen incertidumbres, ya que el verdadero valor de una magnitud depende de las estimaciones o aproximaciones que, a su vez, dependen de la impericia del observador. En las mediciones se identifican tres elementos: número, unidad e incertidumbre, valores asignados mediante varias técnicas.

La precisión de un instrumento es la desviación de los datos que se obtienen en un ensayo, y depende de la naturaleza y el tamaño de la muestra. La exactitud trata de medir el sesgo (bias) de la observación de varias repeticiones, este puede ser sistemático o aleatorio. La sensibilidad depende del indicador del instrumento y el procedimiento, esto indica su confiabilidad (Forsythe, 1985).

Para el análisis de datos experimentales, Vidaurre Garayo (2008) expresa que se consideran tres definiciones, incertidumbre en las medidas, precisión y error. La incertidumbre aparece cuando repetimos una medida experimental varias veces el cual es inherente. La precisión depende del aparato de medición y el error es la diferencia entre el valor observado o calculado y el valor verdadero, el error proviene de varias causas. El error absoluto $\varepsilon(x)$ indica el valor de la incertidumbre en un resultado experimental, se expresa como $(x \pm \varepsilon(x))$ mantiene las misma unidades, el error relativo es adimensional y se expresa como $\varepsilon_{r}(x)=\frac{\varepsilon(x)}{x}$. Al realizar varias mediciones $x_{i}$ el error absoluto correspondiente a cada medición es inicialmente la precisión del aparato $\varepsilon_{p}$, las mediciones se 
pueden expresar como $\left(x_{i} \pm \varepsilon_{p}\right)$. Los errores aleatorios se definen como el error estadístico y es la desviación típica de las medidas, representada en siguiente ecuación.

$$
\sigma=\sqrt[2]{\frac{\sum\left(x_{i}-\bar{x}\right)^{2}}{n}}
$$

Al realizar experimentos de física se presentan dos tipos de mediciones: directa en indirecta. La primera se obtiene al utilizar un instrumento de medida, la medición indirecta ocurre cuando es necesario emplear una expresión matemática que utiliza otras cantidades físicas medidas de forma directa. La determinación de la incertidumbre en medidas indirectas es un proceso que involucra aspectos de cálculo diferencial, debido a que es inevitable la presencia de correlaciones entre las variables de entrada (Cano, Medina, \& Llamosa, 2011).

Para encontrar la propagación del error en mediciones indirectas se puede usar el desarrollo en serie de Taylor para la función $R=f(x, y, z)$ considerando solo términos de primer orden, la incertidumbre bien dada por:

$$
\Delta R=\left|\frac{\partial R}{\partial x}\right| \Delta x+\left|\frac{\partial R}{\partial y}\right| \Delta y+\left|\frac{\partial R}{\partial z}\right| \Delta z
$$

La que coincide con la bien conocida fórmula de la propagación del error y utilizada en la práctica cotidiana de laboratorio para estimar las variables que contribuyen al error que se asigna a la magnitud deseada (Delfina Galles, 1985).

El procedimiento para evaluar la incertidumbre depende de los datos disponibles en la medición directa. Se pueden clasificar dos etapas, primero encontrar las fuentes de incertidumbre, lo que depende de la especificación del equipo, y luego se elabora un plan para la obtención de datos y experimentos adicionales para el estudio de contribuciones simples a la incertidumbre.

Se distinguen dos métodos para cuantificar fuentes de incertidumbre: el método de evaluación Tipo A se basa en el análisis estadístico de las mediciones. El método de evaluación Tipo B comprende otras maneras de estimar la incertidumbre, basado en la experiencia o información externa disponible, asumiendo una función de distribución (Sáen Ruiz \& Font Avila, 2001). 


\section{EXPERIMENTOS}

Los experimentos se realizaron utilizando un plano inclinado con poca fricción, para el análisis de movimiento de los cuerpos se utilizaron las ecuaciones de cinemática deducidas del cálculo (Serway \& Jewett, 2008).

Posición final: $[1] x_{f}=x_{i}+v_{i} t+\frac{1}{2} a t^{2}$

$$
\text { Velocidad final: [2] } v_{f}=v_{i}+a t \quad \text { [3] } v_{f}^{2}=v_{i}^{2}+2 a d
$$

\subsection{VEHÍCULO SOBRE RIEL}

Todos los experimentos partieron del reposo, como se muestra en el Gráfico 1, manteniendo el mismo recorrido en todas las pruebas, se utilizaron sensores magnéticos separados una distancia $\Delta x=x_{f}-x_{i}=1.20 m$. Tras un análisis se derivan las ecuaciones para el cálculo de aceleración y velocidad:

$$
\text { [4] } a=\frac{2 x}{t^{2}} \quad \text { [5] } v_{f}=a t
$$
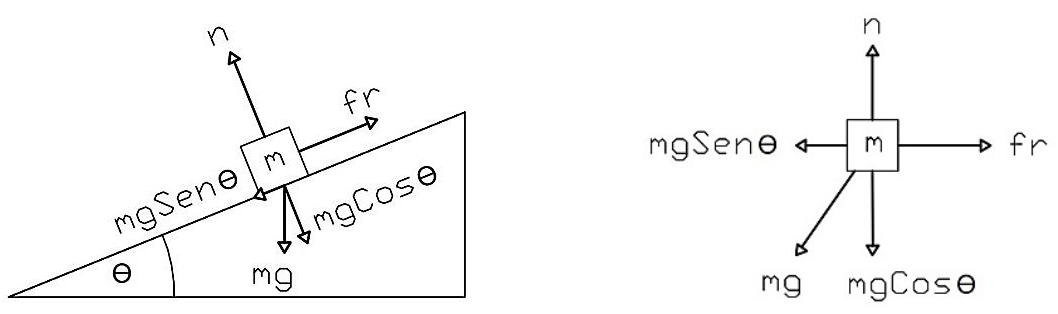

Gráfico 1. Diagrama del cuerpo libre en el plano inclinado. Fuente: Elaboración propia

El plano inclinado también puede utilizarse en experimentos de dinámica, basados en las leyes de Newton. Los bloques no estaban en contacto directo con la superficie, se utilizó un vehículo montado sobre un riel para minimizar la fuerza de fricción, las ecuaciones deducidas fueron las siguientes: 
Fuerza normal: [6] $n=m g \cos \theta$

Fuerza de Fricción: [7] $f_{r}=m(g \sin \theta-a)$

\subsection{BLOQUES UNIDOS CON UNA CUERDA}

Se utilizaron dos bloques unidos a una cuerda, uno de ellos en contacto con el plano inclinado y el otro suspendido en la cuerda como se muestra en el Gráfico 2. La tensión en la cuerda y la fuerza de rozamiento fueron los parámetros a medir. El bloque inició desde el punto más bajo y finalizó en el punto cercano a la polea. Se consideró que los cuerpos parten del reposo y no existe fricción en la polea para realiza los cálculos. Se utilizó el valor de la aceleración obtenido con la ecuación [4].
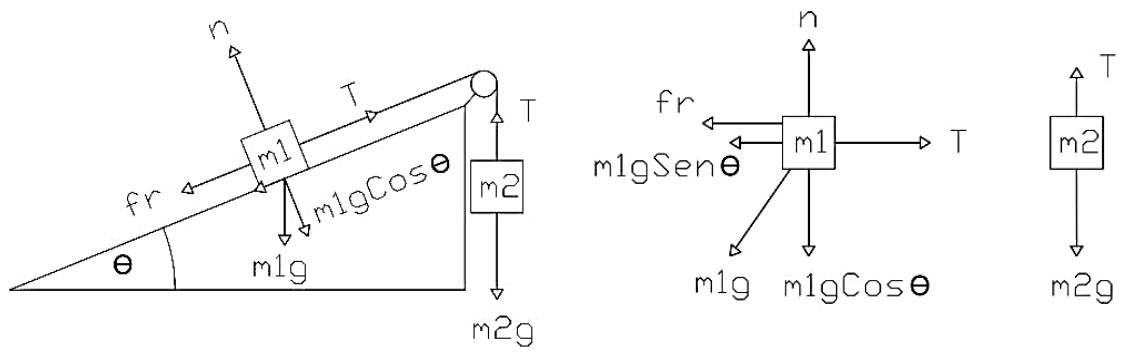

Gráfico 2. Diagrama del cuerpo libre de dos bloques unidos a una cuerda en un plano inclinado. Fuente: Elaboración propia.

Velocidad final: $[8] v_{f}=\sqrt{2 a d}$

Tensión en la cuerda: [9] $T=m_{2}(g-a)$

Fuerza de Fricción: [10] $f_{r}=T-m_{1}(g \sin \theta+a)$ 


\section{DISEÑO MECÁNICO}

La mesa de pruebas consta de un plano inclinado, un vehículo y un sistema de bloques. Para su diseño se utilizó una superficie lisa compuesta por rieles de aluminio. Se consideraron 3 diferentes ángulos de inclinación $10^{\circ}, 15^{\circ}$ y $20^{\circ}$.

\subsection{DISPOSITIVO DE MEDICIÓN}

El dispositivo de medición utiliza la plataforma libre Arduino, contiene un visor, teclas selectoras y dos sensores de luz infrarrojo. Su funcionamiento es sencillo, solo toma la posición inicial de la partícula para empezar el conteo y un sensor en la posición final detiene el contador. Con los tiempos de cada experimento y el ángulo de inclinación, el controlador realiza todos los cálculos necesarios en base a las ecuaciones descritas anteriormente.

\subsection{GRÁFICA DE SEÑALES}

Para la realizar las gráficas del movimiento en función del tiempo se desarrolló una aplicación en Java, la que permitió conectarse vía serial con la plataforma de hardware libre Arduino. Este microcontrolador monitorea el tiempo y realiza los cálculos en base a las ecuaciones descritas anteriormente, a partir de las ecuaciones [2] y [4] el software crea los diagramas de VelocidadTiempo y Aceleración-Tiempo, como se muestra en el Gráfico 3.
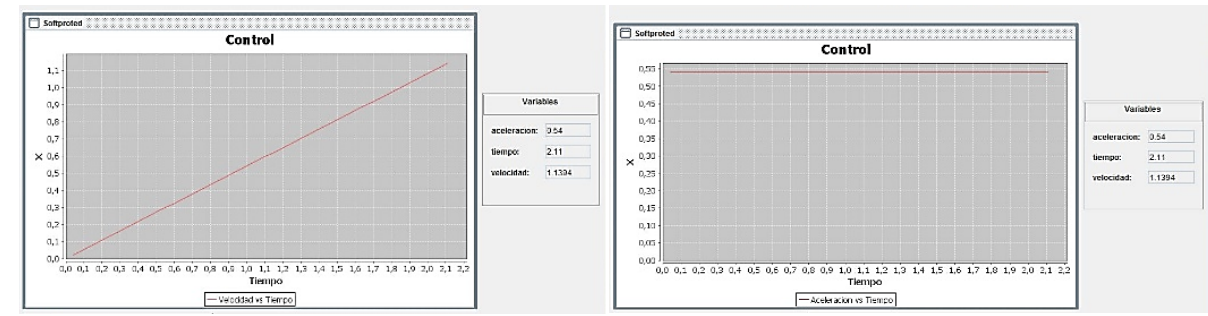

Gráfico 3. Gráficas de Velocidad-Tiempo y Aceleración-Tiempo, del software desarrollado.

Fuente: Elaboración propia 


\section{RESULTADOS}

Las mediciones se realizaron en el plano inclinado, el que cuenta con un visor con cuatro cifras significativas para la medición del tiempo. Con ello se establecieron los cálculos correspondientes a velocidad, aceleración, fuerza de fricción y normal. La medición del tiempo se realizó de manera automática, el contador detecta el paso del vehículo desde la línea de partida utilizando sensores magnéticos ubicados dentro del riel.

\subsection{MEDICIÓN Y CÁLCULO DE ERROR}

La distancia utilizada para los cálculos es $x=0.704 \pm 0.001[m]$, y el error se estima con mínimo valor de la herramienta de medida. Esto depende de la ubicación de los sensores en el riel, los ángulos de inclinación establecidos en el dispositivo son $10^{\circ}, 20^{\circ}$ y $25^{\circ}$, utilizados para el estudio de la incertidumbre.

La Tabla 1 y 2 muestran los valores medidos de tiempo para el desplazamiento del vehículo en el riel con $15^{\circ}$ y $20^{\circ}$ de inclinación respectivamente, los valores de aceleración, velocidad final y fricción se calculan a partir de las ecuaciones [4], [5], [6] y [7]. El valor de la desviación estándar para una población se puede considerar como el error absoluto del tiempo, quedando el enunciado de la medición final como $t=\bar{t} \pm \sigma[s]$.

El valor de la desviación estándar de la población se calcula mediante la siguiente expresión $\sigma=\sqrt[2]{\frac{\sum\left(x_{i}-\bar{x}\right)^{2}}{n}}$, donde n representa el número de las mediciones realizadas. 


\begin{tabular}{|c|c|c|c|c|}
\hline$\theta=15^{\circ}$ & $t[s]$ & $a\left[\frac{m}{s^{2}}\right]$ & $F_{r}[N]$ & $v_{f}\left[\frac{m}{s}\right]$ \\
\hline $\mathrm{t} 1$ & 0,791 & 2,250 & 0,021 & 1,780 \\
\hline $\mathrm{t} 2$ & 0,772 & 2,362 & 0,013 & 1,824 \\
\hline $\mathrm{t} 3$ & 0,782 & 2,302 & 0,017 & 1,801 \\
\hline $\mathrm{t} 4$ & 0,759 & 2,444 & 0,007 & 1,855 \\
\hline $\mathrm{t} 5$ & 0,771 & 2,369 & 0,012 & 1,826 \\
\hline $\mathrm{t} 6$ & 0,753 & 2,483 & 0,004 & 1,870 \\
\hline $\mathrm{t} 7$ & 0,768 & 2,387 & 0,011 & 1,833 \\
\hline $\mathrm{t} 8$ & 0,777 & 2,332 & 0,015 & 1,812 \\
\hline $\mathrm{t} 9$ & 0,789 & 2,262 & 0,020 & 1,785 \\
\hline $\mathrm{t} 10$ & 0,800 & 2,200 & 0,024 & $\mathbf{1 , 7 6 0}$ \\
\hline $\bar{X}$ & $\mathbf{0 , 7 7 6}$ & $\mathbf{2 , 3 3 9}$ & $\mathbf{0 , 0 1 4}$ & $\mathbf{1 , 8 1 5}$ \\
\hline$\sigma^{2}$ & $\mathbf{0 , 0 1 4}$ & $\mathbf{0 , 0 8 4}$ & $\mathbf{0 , 0 0 6}$ & $\mathbf{0 , 0 3 2}$ \\
\hline
\end{tabular}

Tabla 1. Medición de tiempos del desplazamiento de un móvil,para un ángulo de $15^{\circ}$. 


\begin{tabular}{|c|c|c|c|c|}
\hline$\theta=15^{\circ}$ & $t[S]$ & $a\left[\frac{m}{s^{2}}\right]$ & $F_{r}[N]$ & $v_{f}\left[\frac{m}{s}\right]$ \\
\hline $\mathrm{t} 1$ & 0,639 & 3,448 & $-0,007$ & 2,203 \\
\hline $\mathrm{t} 2$ & 0,623 & 3,628 & $-0,020$ & 2,260 \\
\hline $\mathrm{t} 3$ & 0,632 & 3,525 & $-0,013$ & 2,228 \\
\hline $\mathrm{t} 4$ & 0,636 & 3,481 & $-0,010$ & 2,214 \\
\hline $\mathrm{t} 5$ & 0,632 & 3,525 & $-0,013$ & 2,228 \\
\hline $\mathrm{t} 6$ & 0,637 & 3,470 & $-0,009$ & 2,210 \\
\hline $\mathrm{t} 7$ & 0,636 & 3,481 & $-0,010$ & 2,214 \\
\hline $\mathrm{t} 8$ & 0,658 & 3,252 & 0,007 & 2,140 \\
\hline $\mathrm{t} 9$ & 0,642 & 3,416 & $-0,005$ & 2,193 \\
\hline $\mathrm{t} 10$ & 0,649 & 3,343 & 0,001 & 2,169 \\
\hline $\bar{X}$ & $\mathbf{0 , 6 3 8}$ & $\mathbf{3 , 4 5 7}$ & $\mathbf{- 0 , 0 0 8}$ & $\mathbf{2 , 2 0 6}$ \\
\hline$\sigma^{2}$ & $\mathbf{0 , 0 0 9}$ & $\mathbf{0 , 0 9 8}$ & $\mathbf{0 , 0 0 7}$ & $\mathbf{0 , 0 3 1}$ \\
\hline
\end{tabular}

Tabla 2. Medición de tiempos del desplazamiento de un móvil, para un ángulo de $20^{\circ}$.

Se puede observar la estabilidad en las mediciones de correspondientes a $10^{\circ}$ y $20^{\circ}$ respectivamente. El Gráfico 4 contiene las mediciones para un ángulo de $20^{\circ}$, lo que demuestra errores considerables en la medición, por ello se descartó esta opción para su estudio.
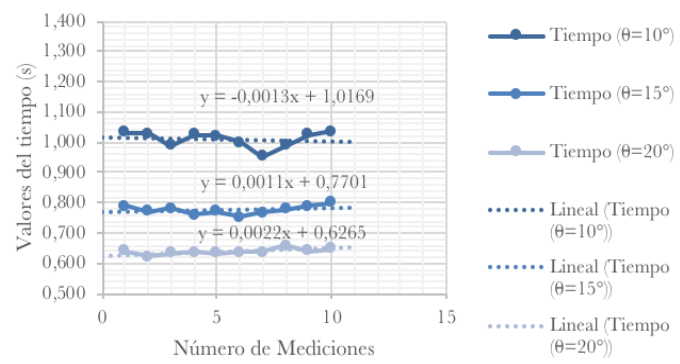

Gráfico 4. Valores de tiempo para $10^{\circ}, 15^{\circ}$ y $20^{\circ}$, incluye líneas de tendencia, ecuación aproximada del gráfico. Fuente: Elaboración propia. 
Los resultados muestran un tiempo estimado de $t_{15}=0.776 \pm 0.014[s]$ para un ángulo de inclinación de $15^{\circ}$ y para $20^{\circ}$ se estima un tiempo de $t_{20}=0.638 \pm 0.09[s]$. El Gráfico 5 presenta valores muy cercanos en la para experimentos a $20^{\circ}$ de inclinación, de la misma manera se presenta poca dispersión en los valores de tiempo tomados a $15^{\circ}$. Los tiempos medidos para experimentos con $10^{\circ}$ de inclinación presentan una mayor incertidumbre $t_{10}=1.010 \pm 0.026[\mathrm{~s}]$.

Lo valores de velocidad en función del tiempo se muestran en el Gráfico 5, ese observa la dispersión de los datos, manteniéndose constante para valores de inclinación superiores a $15^{\circ}$

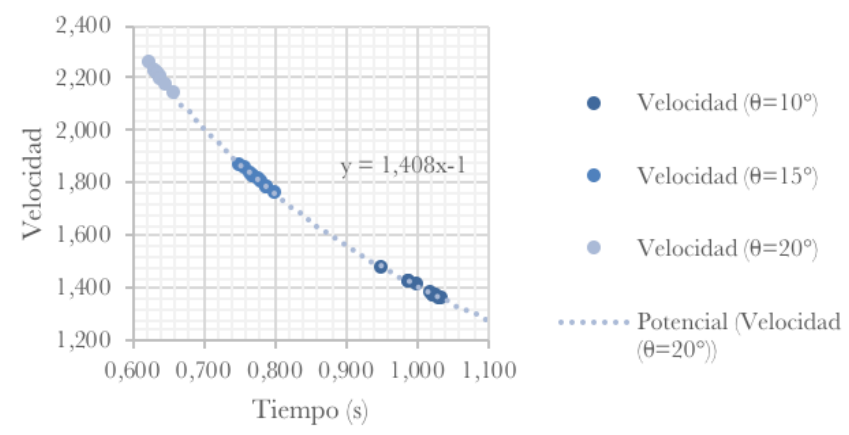

Gráfico 5. Valores de velocidad para $10^{\circ}, 15^{\circ}$ y $20^{\circ}$, incluye curva de tendencia potencial, ecuación aproximada del gráfico.

\subsection{CÁLCULO DE LA INCERTIDUMBRE PARA MEDICIONES INDIRECTAS}

Partiendo las mediciones realizadas en el plano inclinado a $15^{\circ}$ se realizaron cálculos, los cuales presentan una dispersión estable en sus valores a una distancia de $x=0.704 \pm 0.001[m]$. El análisis de las incertidumbres indirectas se realizó considerando el valor medio del tiempo y su error absoluto, aplicando la regla de las derivadas parciales para el cálculo de la incertidumbre. Para $t=0.776 \pm 0.0014[s]$ se obtienen los siguientes resultados:

$$
a=\frac{2 x}{t^{2}}
$$




$$
\begin{gathered}
\Delta a=\left|\frac{\partial a}{\partial t}\right| \Delta t+\left|\frac{\partial a}{\partial x}\right| \Delta x \\
\Delta a=\left|\frac{-4 x}{t^{3}}\right| \Delta t+\left|\frac{2}{t^{2}}\right| \Delta x \\
\Delta a=\left|\frac{-4(0.704)}{0.776^{3}}\right| 0.0014+\left|\frac{2}{0.776^{2}}\right| 0.001 \\
\Delta a=0.088\left[\frac{m}{s^{2}}\right]
\end{gathered}
$$

Se determina la aceleración con la ecuación:

$$
\begin{gathered}
a=\frac{2 x}{t^{2}} \\
a=\frac{2(0.704)}{0.776^{2}}
\end{gathered}
$$

Se puede expresar la aceleración en función de su valor calculado y la incertidumbre obtenida, llamado también error absoluto $a=2.338 \pm 0.088\left[\frac{m}{s^{2}}\right]$. Para la velocidad y la fuerza de fricción se
utilizaron las ecuaciones siguientes:

$$
\begin{gathered}
v_{f}=a t \\
\Delta v=\left|\frac{\partial v}{\partial a}\right| \Delta a+\left|\frac{\partial v}{\partial t}\right| \Delta t \\
\Delta v=|t| \Delta a+|a| \Delta t \\
\Delta v=|0.776| 0.088+|2.338| 0.014 \\
\Delta v=0.101\left[\frac{m}{s}\right]
\end{gathered}
$$


Se puede expresar la velocidad en función de su valor calculado y la incertidumbre obtenida $v=1.814 \pm 0.101\left[\frac{m}{s}\right]$. Finalmente se busca la incertidumbre para la fuerza de fricción, para ello se utilizan los valores en radianes:

$$
\begin{gathered}
f_{r}=m g \sin \theta-m a \\
\Delta f=\left|\frac{\partial f}{\partial \theta}\right| \Delta \theta+\left|\frac{\partial f}{\partial a}\right| \Delta a \\
\Delta f=|m g \sin \theta| \Delta \theta+|-m| \Delta a \\
\Delta f=|0.776| 0.088+|2.338| 0.014 \\
f_{r}=0.014 \pm 0.017[N]
\end{gathered}
$$

$\mathrm{Al}$ expresar la velocidad en función de su valor calculado y la incertidumbre obtenida, se puede asumir que la fricción entre el vehículo y la superficie es muy pequeña. Su error absoluto se expresa como $f_{r}=0.014 \pm 0.017[N]$.

\subsection{ANÁLISIS DE LA INCERTIDUMBRE}

Desde el punto de vista estadístico, considerado el método de evaluación Tipo A, se puede predecir que el valor de la incertidumbre de un instrumento de medida esté inmerso en un intervalo de confianza que garantice que, si se realizan nuevas mediciones, la probabilidad de que este valor se encuentre dentro de ese intervalo sea del 90, 95 o 99\%. Para encontrar la incertidumbre estándar se puede dividir el valor de la incertidumbre calculada para el factor $t$ de Student, siempre y cuando, los datos considerados tengan una distribución normal (Llamosa, Gómez E., \& Ramírez B., 2009).

Para sustentar que la variable independiente: tiempo (en mediciones con $\theta=15^{\circ}$ y $\theta=20^{\circ}$ se distribuye normalmente, se empleó la prueba de Shapiro-Wilk, considerando que el tamaño de las muestras es menor a 30 y asumiendo un nivel de significancia (a) igual a 0,05. (nivel de confianza del 95\%). Se hizo el siguiente planteamiento: 
Hipótesis nula $(\mathrm{Ho})=$ Los datos provienen de una distribución normal.

Hipótesis alterna $(\mathrm{Hl})=$ Los datos No provienen de una distribución normal.

Se acepta la hipótesis nula si el valor de probabilidad es $\geq a$; y se rechaza la hipótesis alterna si el valor de probabilidad es $<a$. Para realizar el análisis se empleó la herramienta estadística SPSS, los resultados obtenidos se encuentran en la Tabla 3.

\section{Pruebas de normalidad}

\begin{tabular}{lr|r|r|r|r|r} 
& \multicolumn{3}{c|}{ Kolmogorov-Smirnov } & \multicolumn{3}{c}{ Shapiro-Wilk } \\
& Estadístico & gl & \multicolumn{1}{c}{ Sig. } & Estadístico & gl & \multicolumn{1}{c}{ Sig. } \\
\hline Tiempo $15^{\circ}(\mathrm{s})$ &, 113 & 10 &, $200^{\star}$ &, 983 & 10 &, 978 \\
\hline Tiempo $20^{\circ}(\mathrm{s})$ &, 175 & 10 &, $200^{\star}$ &, 946 & 10 &, 617 \\
\hline
\end{tabular}

*. Esto es un límite inferior de la significación verdadera.

a. Corrección de significación de Lilliefors

Tabla 3. Prueba de normalidad de las mediciones del tiempo.

El valor de probabilidad para las mediciones del tiempo con un ángulo de inclinación de $15^{\circ}$ es 0,978 y es mayor que 0,05 . Por lo tanto, se acepta la hipótesis nula, es decir se considera que los datos se distribuyen normalmente. De igual manera, ocurre con las mediciones del tiempo con un ángulo de inclinación de $20^{\circ}$, lo que quiere decir que las dos muestras tienen una distribución normal. Lo afirmado, se ratifica en los gráficos Cuantil-Cuantil en los que se puede observar cuan cerca está la distribución de cada conjunto de datos con respecto al valor de su media (ver Gráfico 6). 

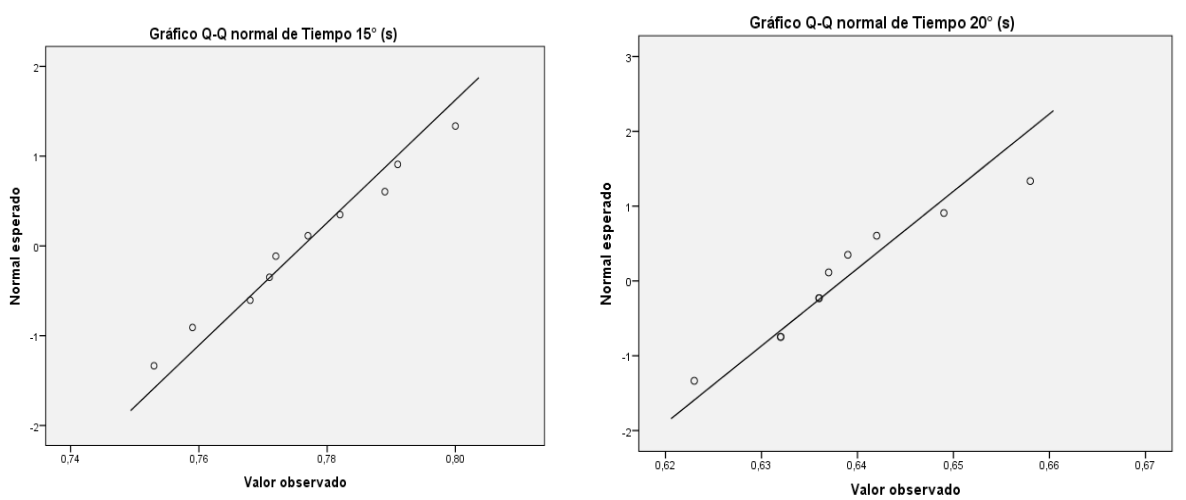

Gráfico 6. Diagramas $Q-Q$ de las mediciones del tiempo $\operatorname{con} \theta=15^{\circ}$ y $\operatorname{con} \theta=20^{\circ}$. Fuente: Elaboración propia.

Con la certeza de que los datos tienen una distribución normal, se puede calcular la incertidumbre expandida.

Realizando un análisis tipo B, podemos considerar como fuentes de incertidumbre el error máximo permisible EMP, la incertidumbre combinada y la ocasionada por varias mediciones. Las ecuaciones utilizadas para su análisis son las siguientes:

$$
\begin{gathered}
E M P=\frac{\text { Clase de exactitud } \cdot \text { Intervalo de medición }}{100} \\
E M P=\frac{1 \cdot 0.800}{100} \\
E M P=0.008[s]
\end{gathered}
$$

La componente de la incertidumbre de varias mediciones se conoce como la varianza, $u_{a}(\bar{m})=s(\bar{m})$. 


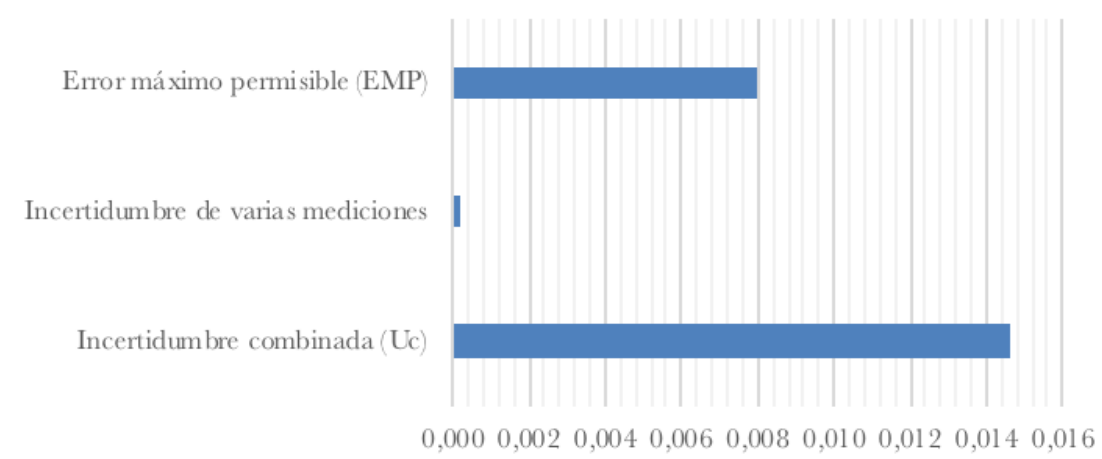

Gráfico 7. Contribuciones a la incertidumbre para una inclinación de $15^{\circ}$.

Fuente: Elaboración propia.

\section{CONCLUSIONES}

La precisión del equipo es 0.001 s valor obtenido de la resolución del instrumento de medida, la incertidumbre en las mediciones se calculó de forma indirecta para los valores de velocidad, aceleración y fuerza de rozamiento. Para el tiempo se realizaron mediciones directas a través de varias pruebas, lo que arrojó un error estadístico de $\pm 0.014[s]$ y error relativo de $1.80 \%$. Estos son los máximos valores obtenidos en las pruebas, Vidaurre Garayo (2008) expresa el análisis de datos experimentales como el máximo valor entre el error del aparato y el error estadístico conocido como el método de evaluación Tipo A.

La fuerza de rozamiento obtenida es pequeña, puesto que esta puede considerarse como despreciable para su estudio en ángulos superiores a $15^{\circ}$. Para ángulos menores que $10^{\circ}$ la fricción no permite el movimiento del vehículo debido a la poca acción de la componente $\mathrm{x}$ del peso, presentando mayor dispersión en los valores, considerado como imprecisión, tal y como lo expresa Forsythe (1985). Estos valores se muestran la figura 3-1. Para la velocidad del vehículo sucede algo similar, ya que mantiene estables sus mediciones y mayor precisión, con un error relativo de $5.56 \%$ para ángulos mayores a $15^{\circ}$. Los valores de tiempo y sus incertidumbres se aproximan considerablemente a las líneas de tendencias presentadas, esto nos permite analizar el comportamiento del vehículo y sus 
errores relativos para mediciones directas a diferentes inclinaciones. Esto se basó en el método de los mínimos cuadrados caracterizado por la ecuación de regresión $y=m x+b$.

Para ángulos menores que $10^{\circ}$ la fricción no permite el movimiento del vehículo debido a la poca acción de la componente $\mathrm{x}$ del peso, presentando mayor dispersión en los valores.

También se utilizó la evaluación de Tipo B de la incertidumbre estándar, asumiendo una función de distribución normal al conocer los valores próximos al centro del intervalo cuyas desviaciones se determinaron por $\frac{a}{\sqrt{3}}$, donde a determina los límites de la incertidumbre. 


\section{REFERENCIAS BIBLIOGRÁFICAS}

Cano, J. A., Medina, M. H., \& Llamosa, L. E. (2011). Metodología para la enseñanza de la estimación de la incertidumbre de medición en experimentos de física. Scientia et Technica Año XVI(49), 207-211. Obtenido de: http://revistas.utp.edu.co/index.php/revistaciencia/article/viewFile/1521/1021

Delfina Galles, C. (1985). Sobre la propagación del error en mediciones indirectas. Revisla Mexicana de Física 32, 169-173. Obtenido de: http://rmf.fciencias.unam.mx/pdf/rmf/32/1/32_1_169.pdf

Forsythe, W. (1985). Manual de Laboratório Física de los Suelos. San José, Costa Rica: IICA. Obtenido de: https: / /books, google.es/books?id=mfM-ih0OgIIC\&dq=incertidumbre+laboratorio+de+fisica\&l$\mathrm{r}=\& \mathrm{hl}=\mathrm{es} \&$ source $=\mathrm{gbs} \_$navlinks_s

Franco García, A. (2017). Errores en las medidas. Obtenido de: (Física con ordenador): http://www. sc.ehu.es/sbweb/fisica/unidades/medidas/medidas.htm

Llamosa, L., Gómez E., J., \& Ramírez B., A. (2009). Metodología para la estimación de la incertidumbre en mediciones. Scientia et Technica, XV(41), 384-389.

Pontes, A. (2006). Diseño y aplicación educativa de un programa de simulación para el aprendizaje de técnicas experimentales con sistemas de adquisición de datos. Revista Eureka sobre Enseñanza y Divulgación de las Ciencias.

Sáen Ruiz, S., \& Font Avila, L. (2001). Incertidumbre de la Medición a la Práctica. Maracay, Estado Aragua: L\&S Consultores.

Schmid, W., \& Lazos Martínez , R. (2004). Guía para estimar la incetidumbre de la medición. México: El Marqués. Obtenido de: http://publicaciones.ops.org.ar/publicaciones/cursos_virtuales/OAA/Material $/ \mathrm{Gu} \% \mathrm{C} 3 \% \mathrm{ADa} \% 20$ para $\% 20$ estimar $\% 20$ incertidumbre.pdf

Serway, R., \& Jewett, J. (2008). Física para Ciencias e Ingeniería. Mexico: Cengage Learning.

U.L.P.G.G. (2017). Análisis de datos y teoria de errores. Obtenido de: Prácticas de Física de la Escuela Universitaria de Ingeniería Técnica de Telecomunicaciones U.L.P.G.C. : https://www2.ulpgc.es/hege/almacen/download/7/7464/Practica_1.pdf

Vidaurre Garayo, A. J. (2008). Análisis de datos experimentales. Valencia, España. Obtenido de: http://hdl.handle.net/10251/1538 